\title{
Live Multicellular Tumor Spheroid Models For High-Content Imaging and Screening In Cancer Drug Discovery
}

\author{
Brian G. Reid ${ }^{1}$, Taleen Jerjian ${ }^{1}$, Purvi Patel ${ }^{2}$, Qiong Zhou ${ }^{1}$, Byong Hoon Yoo ${ }^{1}$, Peter Kabos ${ }^{3}$, Carol \\ A. Sartorius ${ }^{2}$ and Daniel V. LaBarbera ${ }^{1, *}$ \\ ${ }^{I}$ Department of Pharmaceutical Sciences, Skaggs School of Pharmacy and Pharmaceutical Sciences, and Departments \\ of ${ }^{2}$ Pathology and ${ }^{3}$ Medical Oncology, School of Medicine, The University of Colorado, Anschutz Medical Campus, \\ Aurora CO, USA
}

\begin{abstract}
The multi cellular tumor spheroid (MCTS) model has been used for decades with proven superiority over monolayer cell culture models at recapitulating in vivo tumor growth. Yet its use in high-throughput drug discovery has been limited, particularly with image based screening, due to practical and technical hurdles. Here we report a significant advance in utilizing live MCTS models for high-content image based drug discovery. Using a validated GFP reporter (CK5Pro-GFP) of luminal breast cancer stem cells (CSC), we developed an algorithm to quantify changes in CK5ProGFP expression levels for individual Z-stack planes (local) or as maximal projections of the summed Z-stacks (global) of MCTS. From these image sets, we can quantify the cross-sectional area of GFP positive cells, the fluorescence intensity of the GFP positive cells, and the percent of spheroid cross-sectional area that expresses CK5Pro-GFP.We demonstrate that acquiring data in this manner can be done in real time and is statistically robust $\left(Z^{\prime}=0.85\right)$ for use in primary high-content screening cancer drug discovery.
\end{abstract}

Keywords: Multicellular Tumor Spheroid (MCTS), High-Throughput Screening (HTS), High-Content Screening (HCS), Cancer Stem Cells (CSC), Cytokeratin 5, "Systems Biology", Luminal Breast Cancer, and Cancer Drug Discovery.

\section{INTRODUCTION}

It has been forty-three years since Sutherland and coworkers first described the in vitro $3 \mathrm{D}$ tumor model, now referred to as the multicellular tumor spheroid (MCTS) [1, 2]. From this very first report and subsequent articles the MCTS model has displayed properties that significantly differ from monolayer (2D) cancer cell culture; notably a morphology that closely resembles in vivo human tumor growth and resistance to conventional chemotherapy and radiation [1, 3-10]. Accordingly, it is well accepted that the MCTS model may be useful in bridging the gap of information that exists between 2D-cell culture models and in vivo models $[11,12]$. Indeed, MCTS models are highly amenable to heterotypic cell culture, and have played an important role in characterizing the function of the microenvironment in tumor progression as well as promoting invasion and metastasis [13-16]. When compared to 2D-cell culture models, multiple studies have demonstrated that $3 \mathrm{D}$ cell culture models better recapitulate in vivo tumor gene expression profiles [17, 18]. Furthermore, the MCTS appears to be ideal for studying subsets of cells that exist in heterogeneous tumors in vitro, particularly those cells that can self renew, initiate tumor

*Address correspondence to this author at the Department of Pharmaceutical Sciences, The Skaggs School of Pharmacy and Pharmaceutical Sciences, The University of Colorado, Anschutz Medical Campus, 12850 East Montview Blvd, Room V20-2101, Aurora, CO 80045, USA; Tel: (303)724-4116; Fax: (303)724-7266; E-mail: daniel.labarbera@ucdenver.edu, growth, resist cell death, and can promote tumor recurrence and metastasis, termed herein as cancer stem cells (CSCs) [19-21]. Hence, the MCTS may also be an ideal model for drug discovery and development targeting CSCs.

Advances in technology and methods to culture MCTS suitable for high-throughput screening (HTS) and more recently image-based high-content analysis have generated a surge of interest in utilizing the MCTS model for novel drug discovery. Recently, we reviewed this topic and refer the reader to this article for a comprehensive overview [5]. In addition to its superiority over 2D-cell based models in mimicking in vivo tumor growth, the MCTS model offers a "systems biology" $[22,23]$ approach to cancer drug discovery in the context of the tumor microenvironment packaged in one assay. For example, the tumor microenvironment has a role in regulating and promoting cell signaling, gene expression, and overall tumor morphology including invasion and metastasis. If we consider the spheroid as a tumor and the extracellular matrix(ECM) as part of the microenvironment then together this could be considered a basic system. Incorporating heterotypic cell culture and other components of the microenvironment would expand such systems in the future. Heterotypic MCTS culture methods are adaptable to highthroughput technologies and screening platforms including image-based high-content screening (HCS) [24-28]. Nevertheless, significant hurdles exist with HCS data acquisition and automated analysis, limiting MCTS systems to endpoint analysis (e.g. MCTS viability) with no spatiotemporal information. Therefore, developing methods to analyze live 


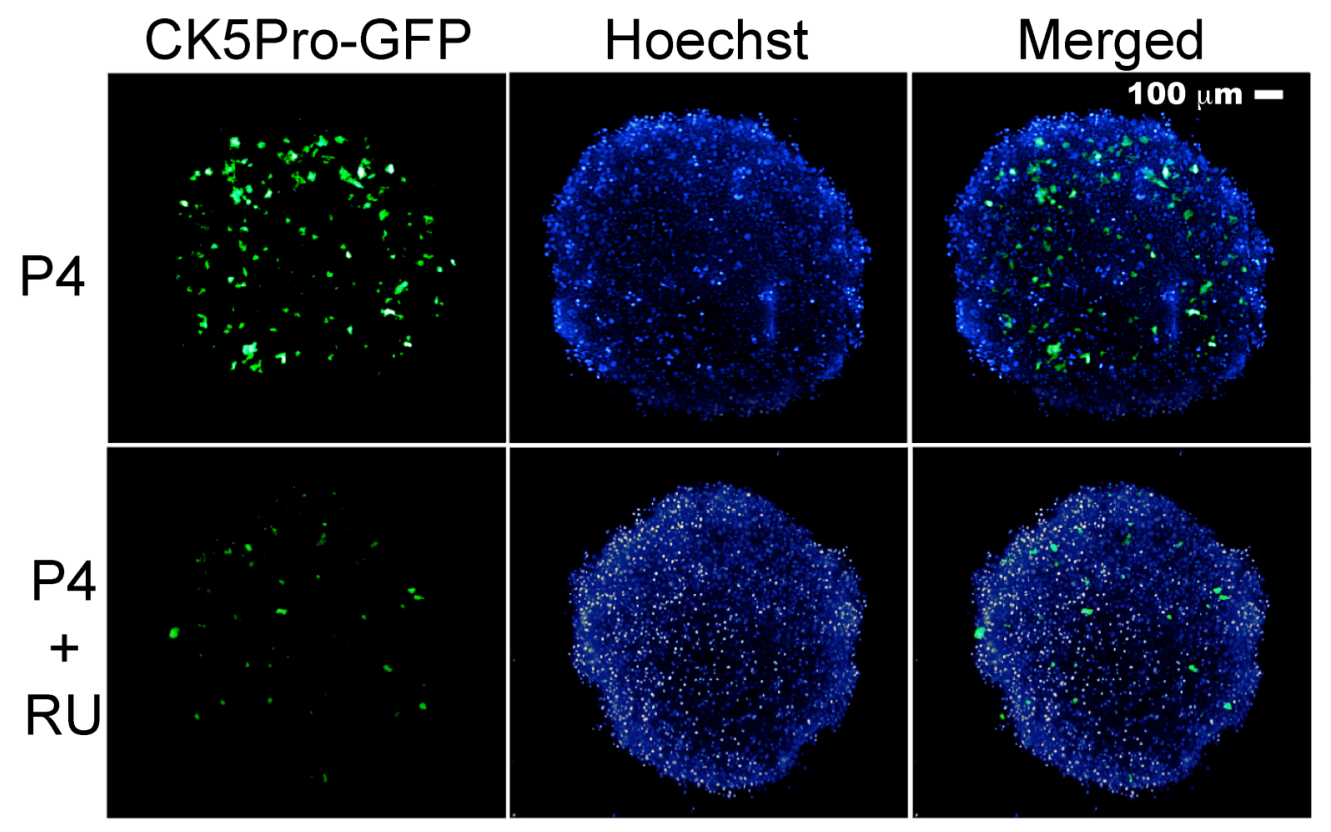

Fig. (1). Live MCTS expressing GFP reporter of luminal breast CSCs imaged in 3D. Maximal Projections of a CK5Pro-GFP-T47D spheroid treated with $100 \mathrm{nM}$ progesterone (P4) over $72 \mathrm{~h}$, resulting in global expansion of the CSC sub-population (top panel). RU486 (RU) $1 \mu \mathrm{M}$ blocks global expansion of this CSC phenotype in CK5Pro-GFP-T47D spheroids (bottom panel). See supporting information for time lapse videos of P4 induced CSC expansion and inhibition using $1 \mu \mathrm{M}$ isotretinoin.

MCTS models locally (within cells and zones of spheroids) and globally (whole spheroids) using automated high-content imaging will effectively advance the MCTS model to be used as an HCS system for cancer drug discovery. To this end, we report a novel MCTS model designed to identify small molecule modulators of a sub population of CSCs existing within a population of luminal breast cancer cells. This model system utilizes cytokeratin 5 (CK5) as a biomarker readout and surrogate reporter for this CSC phenotype in T47D luminal breast cancer cells.

CK5 marks bi-lineage progenitor cells in the normal breast capable of differentiating into luminal or ba$\mathrm{sal} /$ myoepithelial cells $[29,30]$. Increased expression of CK5 is correlated with poor prognosis across all breast cancer subtypes [31]. Poor prognostic basal-like breast cancers, the major subtype of triple negative breast cancer, have the highest $\mathrm{CK}^{+}$cell content $[32,33]$. Many luminal estrogen receptor $(E R)^{+}$breast tumors also contain subpopulations of $\mathrm{CK}^{+}$cells that are enriched in the expression of stem and mesenchymal genes, and are more quiescent, self-renewing, and drug resistant compared to intratumoral $\mathrm{CK} 5^{-}$cells [34, 35]. Furthermore, the $\mathrm{CK}^{+}$cell population in luminal breast cancer expands upon treatment with progesterone (P4), a pro-tumorigenic hormone under some contexts [34-37]. Recently, we validated CK5 expression as a surrogate reporter of this CSC population in T47D luminal breast cancer cells utilizing the CK5 promoter (CK5Pro) enhanced green fluorescent protein (GFP) reporter stably transduced into T47D cells yielding the CK5Pro-GFP-T47D cell line [38]. To validate this reporter for HCS we utilized DMSO as a negative control and RU486, a potent inhibitor of progesterone receptor (PR), as a positive control. RU486 blocks P4 induced expansion and transformation of the CSC phenotype with a Z'factor $\geq 0.50$ per plate tested. Pilot screening with this reporter via a 2D-cell based HCS yielded four hits including three retinoids. Retinoic acid proved to be a potent inhibitor of CK5 expression $\left(\mathrm{IC}_{50}=800 \mathrm{pM}\right)$ and CSC phenotype expansion. We have now adapted this reporter for use with T47D MCTS in 96-well plates, cultured uniformly, and incorporating an extracellular matrix (ECM). We have developed methods to measure and quantitate the modulation of this CSC population using retinoids in live MCTS both locally and globally, and have validated these methods for HCS-based drug discovery.

\section{MATERIALS AND METHODOLOGY}

\section{Materials}

The CK5Pro-GFP-T47D cell line was produced and maintained as previously described [38]. Cell culture reagents and drug compounds were obtained and used as described below or as directed from the manufacturer, including: Dulbecco's Modified Eagle Medium (DMEM) from Corning Life Sciences (Union City, CA, catalogue\#15-013$\mathrm{CM}$ ), phenol free-DMEM from Sigma Aldrich (St. Louis, MO, catalogue\#D1145), HyClone fetal bovine serum (FBS) from Thermo Fisher Scientific (Rochester, NY, catalogue\#SH30071), Matrigel ${ }^{\mathrm{TM}}$ (Bedford, MA) Progesterone (P4) from Sigma Aldrich (St. Louis, MO, catalogue\# P8783), 13-cis-Retinoic acid (isotretinoin) from Sigma Aldrich ( $\mathrm{St}$ Louis, MO, catalogue\# R3255), RU486 from Sigma Aldrich (St Louis, MO, catalogue\# M8046), dimethyl sulfoxide (DMSO) and ethanol (EtOH) from Thermo Fisher Scientific (Rochester, NY, catalogue\# BP231), bisBenzimide Hoechst 33342 trihydrochloride from Sigma Aldrich (St. Louis, MO catalogue\# B2261). Unless otherwise stated below, black 96well microplates were used for imaging and were obtained from Greiner Bio-One (Monroe, NC, catalogue\# 655090). 


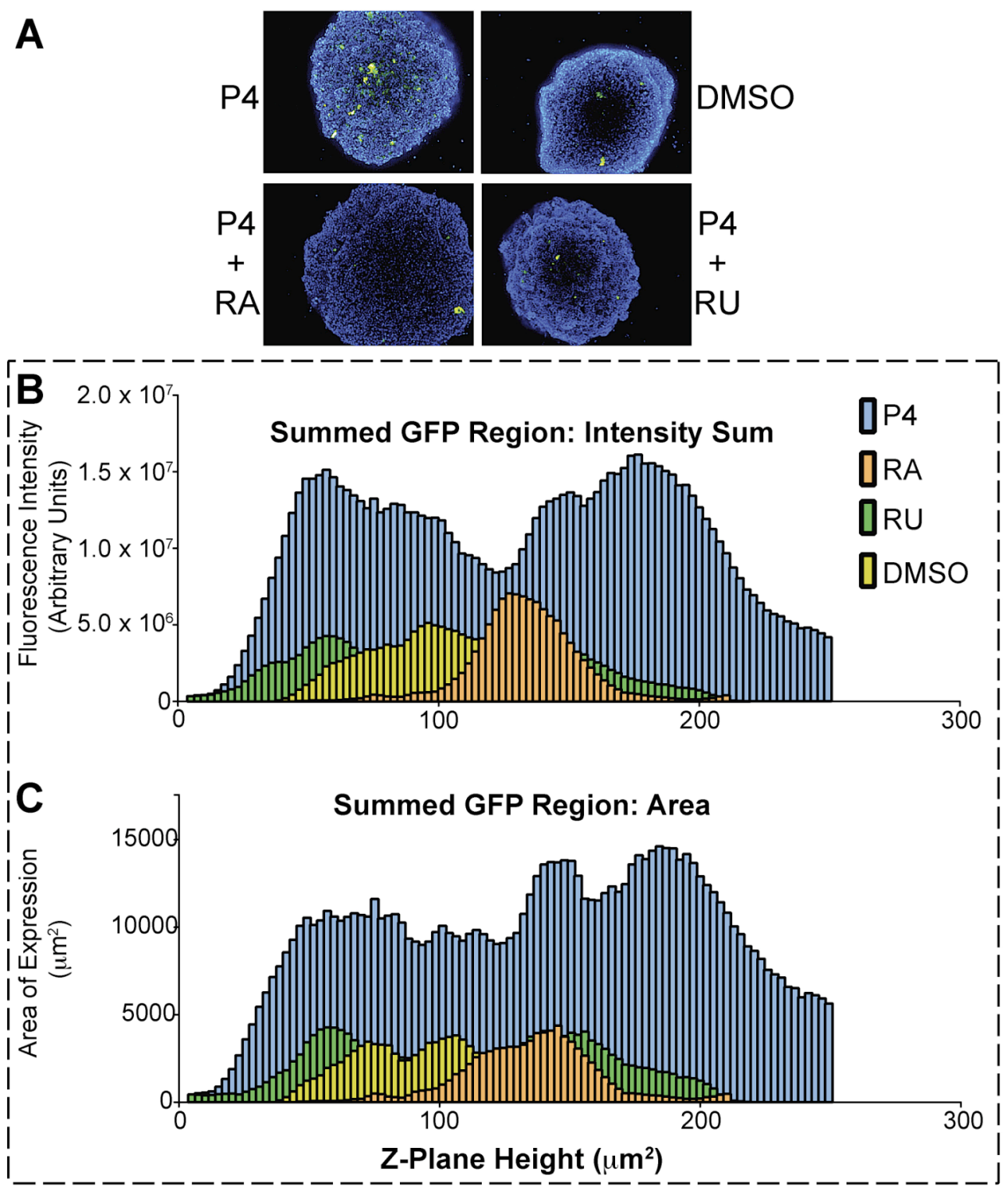

Fig. (2). High content imaging to quantitate the heterogeneity of reporter expression in individual spheroids using all three dimensions. A) Maximal projection images of spheroids where Z-stack images were taken at steps of $2.6 \mu \mathrm{m}$ over a $\mathrm{Z}$ span of $240 \mu \mathrm{m}$. Individual planes were combined in a maximal projection. High content output parameters shown across each focal plane of spheroids shown in B) The sum of fluorescence intensity across each plane, and C) The area of CK5Pro-GFP expression in $\mu^{2}{ }^{2}$. Compound abbreviations are: RU486 $(\mathrm{RU})$, Isotretinoin (RA), progesterone (P4), and DMSO is the vehicle control.

\section{CK5Pro-GFP-T47D MCTS Culture}

Single uniform CK5Pro-GFP-T47D breast carcinoma spheroids were cultured in 96-well microplates using a modified liquid overlay method previously described [6]. Briefly, each well of a 96-well flat bottom plate was coated with $50 \mu \mathrm{L}$ of $1.5 \%$ agarose solution in serum-free and phenol redfree DMEM. During the coating process, the agarose solution was maintained at $\geq 60{ }^{\circ} \mathrm{C}$ followed by cooling and setting at room temperature for $40 \mathrm{~min}$. CK5Pro-GFP-T47D cells were plated at a density of 10,000 cells per well in $50 \mu \mathrm{L}$ phenol red-free DMEM supplemented with $10 \%$ FBS. Next, the plate was centrifuged at $1000 \mathrm{rpm}$ for $15 \mathrm{~min}$ at room temperature to induce cell aggregation. Aggregated cells were coated with $50 \mu \mathrm{L}$ of a $10 \%$ solution of growth factor-reduced Matrigel ${ }^{\mathrm{TM}}$ (protein concentration: 7.1 $\mathrm{mg} / \mathrm{mL}$ ) in phenol red-free DMEM supplemented with $10 \%$ FBS, resulting in a final concentration of 5\% Matrigel ${ }^{\mathrm{TM}}$. After two days of incubation, an additional $50 \mu \mathrm{L}$ of $5 \%$ Matrigel ${ }^{\mathrm{TM}}$ containing medium was added to each well to maintain a $5 \%$ Matrigel $^{\mathrm{TM}}$ concentration in a final volume of $150 \mu \mathrm{L}$ per well. Before treatment, spheroids were cultured for 7 days yielding an average diameter of $1 \mathrm{~mm}$ under standard tissue culture conditions $\left(37^{\circ} \mathrm{C}, 5 \% \mathrm{CO} 2\right)$.

\section{CK5Pro-GFP-T47D MCTS Treatment and Time Lapse- Video Capture}

To generate time-lapse videos, spheroids were transferred into a glass bottom 96-well plate (Whatman, catalogue\# 7716-2375) and treated with vehicle (EtOH), 100nM P4 and $100 \mathrm{nM} \mathrm{P} 4+1 \mu \mathrm{M}$ isotretinoin. Following treatment, spheroid 
images were captured every $15 \mathrm{~min}$ for $12.5 \mathrm{~h}$ using a $3 \mathrm{I}$ MARIANAS inverted Spinning Disk microscope.

\section{CK5Pro-GFP-T47D MCTS Treatment and High-Content Imaging}

Spheroids were treated with $100 \mathrm{nM}$ P4 and varying concentrations of RU486, isotretinoin, 5-fluorouracil (5FU), or Tamoxifen, ranging from $0.001 \mathrm{nM}$ to $10 \mu \mathrm{M}$, over a $72 \mathrm{~h}$ timecourse, using DMSO (0.5\% final volume [38]) as the solvent vehicle. Spheroids were either imaged directly or cell nuclei were stained with $10 \mu \mathrm{M}$ Hoechst 33342 (bisBenzimide $\mathrm{H} 33342$ trihydrochloride), at $37{ }^{\circ} \mathrm{C}$ for $30 \mathrm{~min}$, before live spheroid image based analysis. Spheroids were transferred to 96 well plates along with $50-100 \mu \mathrm{L}$ of media and imaged using an Operetta (Perkin Elmer) equipped with a live-cell chamber that was pre-equilibrated to $5 \% \mathrm{CO}_{2}$ and $37{ }^{\circ} \mathrm{C}$. For detailed global image acquisition, a 10X HighNA objective was used in spinning-disc confocal mode. Zplanes were imaged in $2.6 \mu \mathrm{m}$ steps. For whole-plate imaging, a 10X Long-WD objective was used in spinning-disc confocal mode with $8 \mathrm{Z}$-planes imaged for each field, with $25 \mu \mathrm{m}$ steps between Z-planes.

\section{CK5Pro-GFP MCTS High Content Analysis}

Acquired images were analyzed using Columbus software (Perkin Elmer). Names used for general high content output fields are listed in parentheses below. Analysis steps include region-based image segmentation based on regions of green fluorescence (GFP Region). Selected regions were tiled with spots, and intensity and morphological properties were calculated for all spots. Spots were selected based on spot size $\left(\mathrm{px}^{2}\right)$ and spot intensity in order to filter out nonspheroid related or background selected fluorescence (Selected GFP Region). High content fields were summed across the total area of selected spots to determine the "Summed GFP Region: Intensity Sum" and the "Sum GFP Region: Area" parameters. For the dose-response and Zprime figures, analysis was done on a maximal projection image representing the peak values of each of 8 Z-planes projected onto a single image. For the detailed analysis of whole spheroids, a similar analysis was done using Harmony software (Perkin Elmer), where each Z-plane was analyzed individually.

\section{Statistical Analysis}

Z-primes were calculated using the formula (Zhang et al.) [39]:

$$
Z^{\prime} \text { Factor }=1-\frac{3(\sigma p+\sigma n)}{|\mu p-\mu n|}
$$

Five replicates each of positive controls and negative controls were used. Positive controls shown were $10 \mu \mathrm{M}$ isotretinoin and negative controls included $0.001 \mathrm{nM}$ isotretinoin. For $\mathrm{IC}_{50}$ determinations, each data point is representative of data from five spheroids in a single 96-well plate. Output values from the analyses above were plotted on a semi-log scale and fit to a 4-parameter sigmoidal dose response curve (Graphpad Prism).

\section{RESULTS AND DISCUSSION}

Although the study of MCTS as a model system for tumor biology has been pursued for over forty years, more recent advances in imaging and screening instrumentation, as well as in analytical software, has brought the MCTS to the brink of being a valid primary screening tool for cancer drug discovery. The advantages to image-based primary screening in MCTS are several-fold, and include: the ability to distinguish region specific, local effects of small molecules on MCTS phenotype, and whole-spheroid global effects. This approach can also provide information on small-molecule permeability into biologically relevant tissue models as well as on the toxicity of screening compounds at the primary screening level.

The difficulties involved in primary image-based screening of MCTS should not be underestimated; however, we propose that these are primarily issues of practicality. Here we present an initial pathway to large-scale primary screening of MCTS with an eye towards multiparametric readouts with sub-spheroid spatiotemporal resolution. We first demonstrate that live MCTS can be imaged in an environmentally controlled chamber of a high-content imaging system (Fig. 1). We chose the CK5Pro-GFP-T47D cell line as the MCTS model because of the clinical relevance of CK5 expression in breast tumors [38].

This reporter of the CSC phenotype expresses in only a subpopulation of the cells that comprise the individual MCTS when treated with P4. We can block P4 induced expansion of this CSC phenotype using the potent PR inhibitor RU486. In a previous screen, we identified three retinoids that do not inhibit PR directly but are also potent inhibitors of CK5 expression and expansion of the CSC phenotype [38]. Here, we demonstrate the efficacy of one of these retinoids in MCTS, using time lapse videos of P4 and 13-cisRetinoic acid (isotretinoin) treated spheroids over a $12 \mathrm{~h}$ time course (supplemental material). Individual CK5ProGFP-T47D spheroids were cultured in the presence of $5 \%$ ECM (Matrigel ${ }^{\mathrm{TM}}$ ) to a diameter of $\sim 1 \mathrm{~mm}$. When $\mathrm{P} 4$ is added to individual spheroids there is a significant upregulation of CK5Pro-GFP activity. However, isotretinoin instantly blocks P4 induced CK5 expression preventing expansion of the CSC phenotype over this $12 \mathrm{~h}$ time course. The heterogeneity of CK5 expression in MCTS makes it an excellent candidate for image-based analysis, where spatial resolution of CK5 expression in live spheroids can be examined and quantified in much greater detail than with an endpoint readout such as whole-well luminescence from a luciferase reporter. This will also allow us, when fully validated for screening, to separately analyze individual spheroids should there be multiple spheroids in a single well of a multiwell plate, which will significantly ease the burden of using an automated high-throughput spheroid manipulation approach.

In Fig. (2), we show that our image-based approach is capable of not just observing, but also quantifying changes in CK5Pro-GFP expression in individual Z-planes using intact, live MCTS. The heterogeneity of CK5Pro-GFP expression in the $\mathrm{X}$ and $\mathrm{Y}$ dimensions of each individual plane that we observe by eye is recapitulated in the Z-dimension, as shown quantitatively in the histograms. Furthermore, we show that overall CK5Pro-GFP expression level can be quantified for 

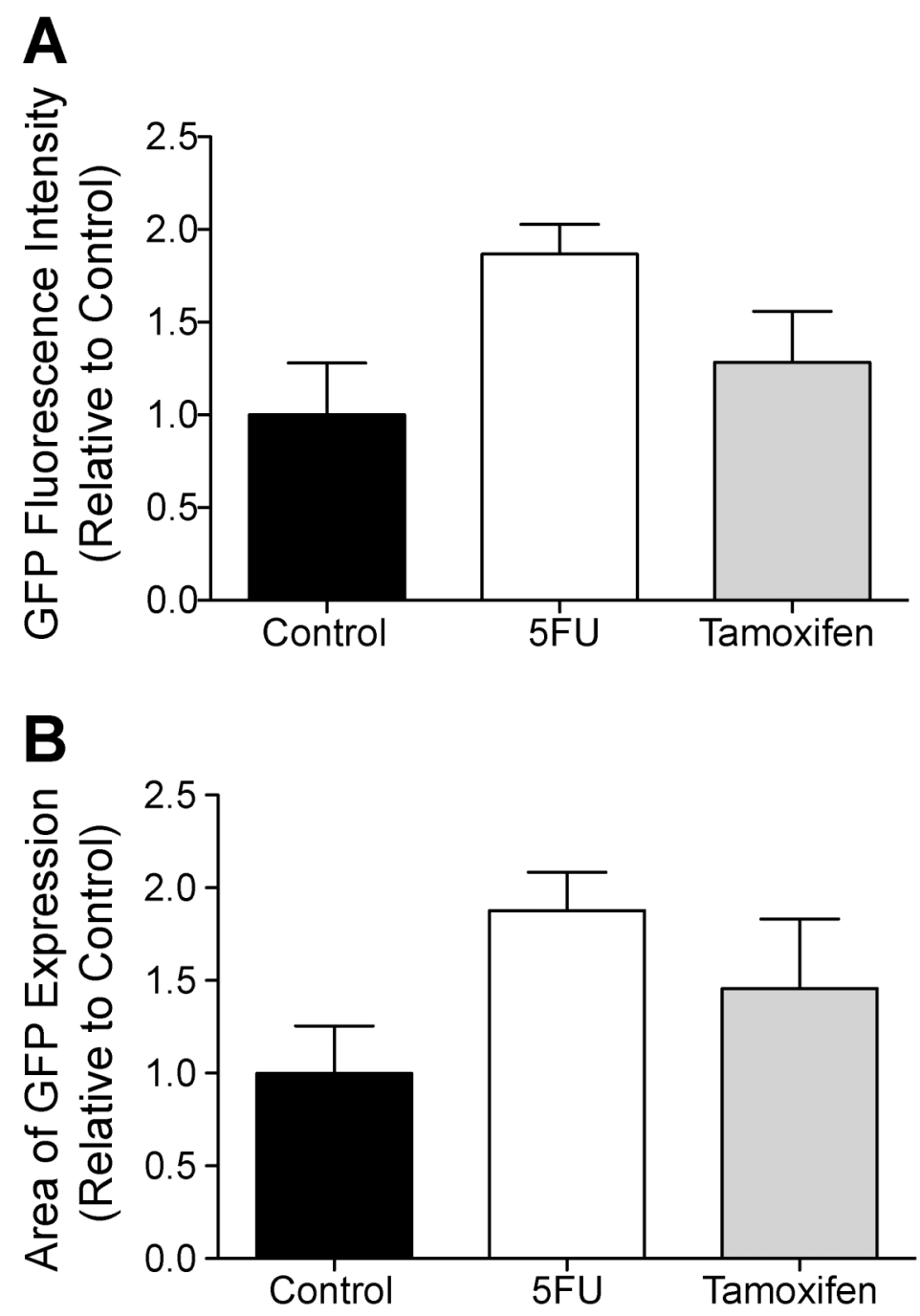

Fig. (3). Validation testing with clinically used cancer drugs. To show that CK5Pro-GFP is a selective reporter for the CSC phenotype we utilized the clinically used cancer drugs 5FU and Tamoxifen. MCTS were treated with $100 \mathrm{nM}$ P4 and $1 \mu \mathrm{M} 5 \mathrm{FU}$ or Tamoxifen for $72 \mathrm{~h}$. The CK5Pro-GFP activity was quantitated using the sum of A) Fluorescence intensity and B) Area of GFP expression. The mean and SEM were obtained using four MCTS per treatment group and the data were normalized to the control group (100 nM P4 + vehicle). These data indicate that CSC phenotype is not downregulated as a result of cytotoxicity caused by 5FU and the estrogen receptor antagonist Tamoxifen.

each individual plane as a distinct parameter. From the same image set, we can quantify the cross-sectional area of GFP positive cells, and the percent of spheroid cross-sectional area that express CK5Pro-GFP. For the compounds tested (RU-486, isotretinoin) we see concomitant decreases in overall CK5Pro-GFP expression and fraction of CK5ProGFP positive cell area.

In a high throughput screen where we can monitor these fields independently using a single image set, we may find compounds that can affect just one of these parameters. For example, one can imagine a compound that decreases the fraction of GFP positive cells (or GFP positive area) while maintaining the average expression levels in the GFP positive area. In a secondary analysis of such a compound, a time course of expression could be done to see whether the effect is due to diffusion rate of a compound through the spheroid or to a particular effect on a biological pathway, although determination of the pathway could require a significant amount of downstream work. Regardless, the point of this theoretical example is that we can monitor many different parameters in live MCTS simultaneously, determine empirically which parameters change in response to treatment with small molecules, and even look for differential effects of compounds on multiple parameters, using a single image set from each well of a multi well plate. Next, to further validate the CK5Pro-GFP activity as a faithful reporter of the CSC phenotype we treated MCTS with the clinically used breast cancer therapies, including: the cytotoxic agent five fluorouracil $(5 \mathrm{FU})$, and the estrogen receptor antagonist Tamoxifen (Fig. 3) [40-42]. Both 5FU and Tamoxifen treatments did not induce down regulation of CK5Pro-GFP expression, indicating that CK5Pro-GFP activity is a specific reporter for progesterone induced CSC phenotype expansion.

Having determined satisfactory image acquisition methods, we went on to write the analysis algorithm. The algorithm was written using a building-block approach in the 


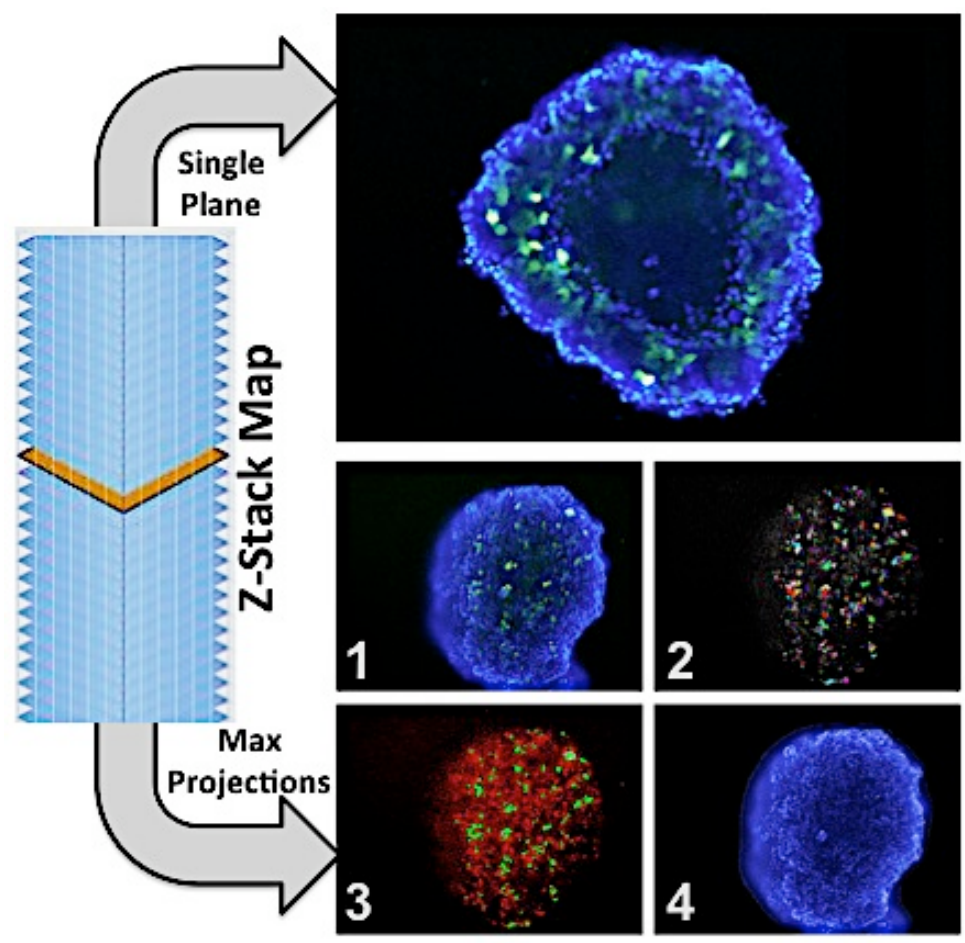

Fig. (4). A robust algorithm can capture both local (single plane) data and global (whole spheroid) phenomena. Using a specific field within a well of a 96-well plate an algorithm for analysis of CK5Pro-GFP expression in MCTS can be used to capture data in a single plane or globally by using maximal projections. Panels 1-4 (bottom right) show the maximal projection of 8 Z-planes. Panel 1 shows the stained nuclei (Hoechst, blue) and the CK5Pro-GFP expression (green). Panel 2 shows the regions of the image tiled with spots (arbitrary colors). Panel 3 shows spots selected by fluorescence intensity and size (selected spots in green, rejected spots in red). Panel 4 shows the entire spheroid region across which high-content parameters were summed.
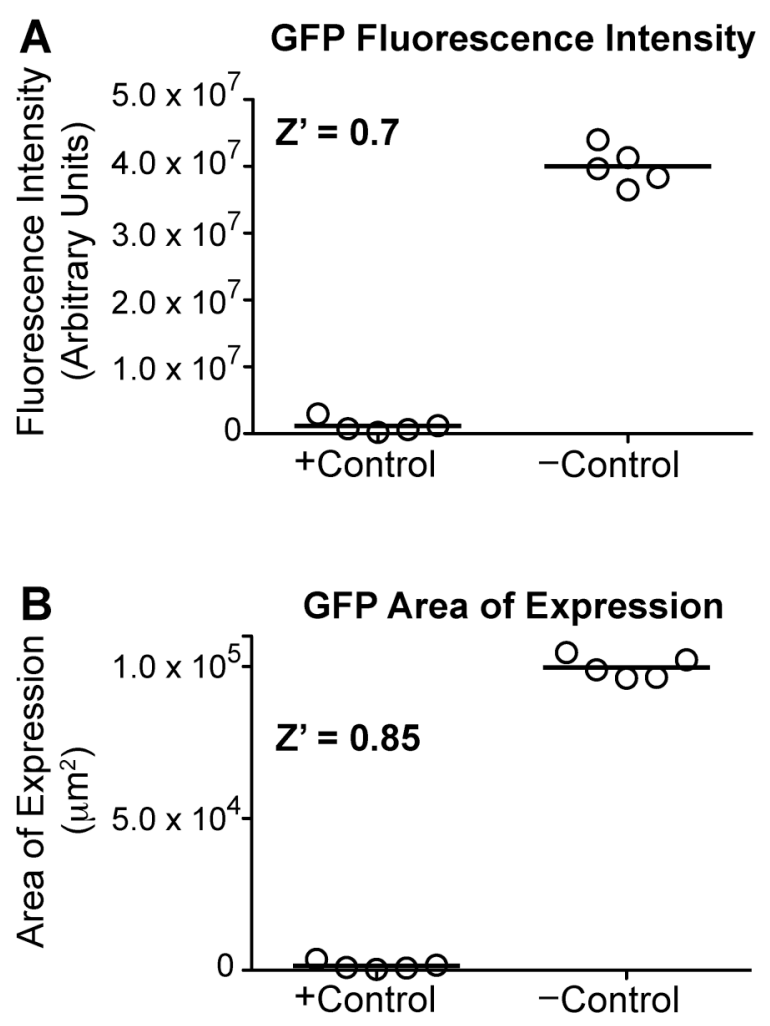

Fig. (5). Z'-Factor analysis showing the robustness of this algorithm and data. Z' prime scores were generated for A) Fluorescence intensity, and B) The area of GFP expression. $Z$ ' scores were determined using 5 positive controls (10 $\mu \mathrm{M}$ isotretinoin) and 5 negative controls (0.001 $\mu \mathrm{M}$ isotretinoin). 

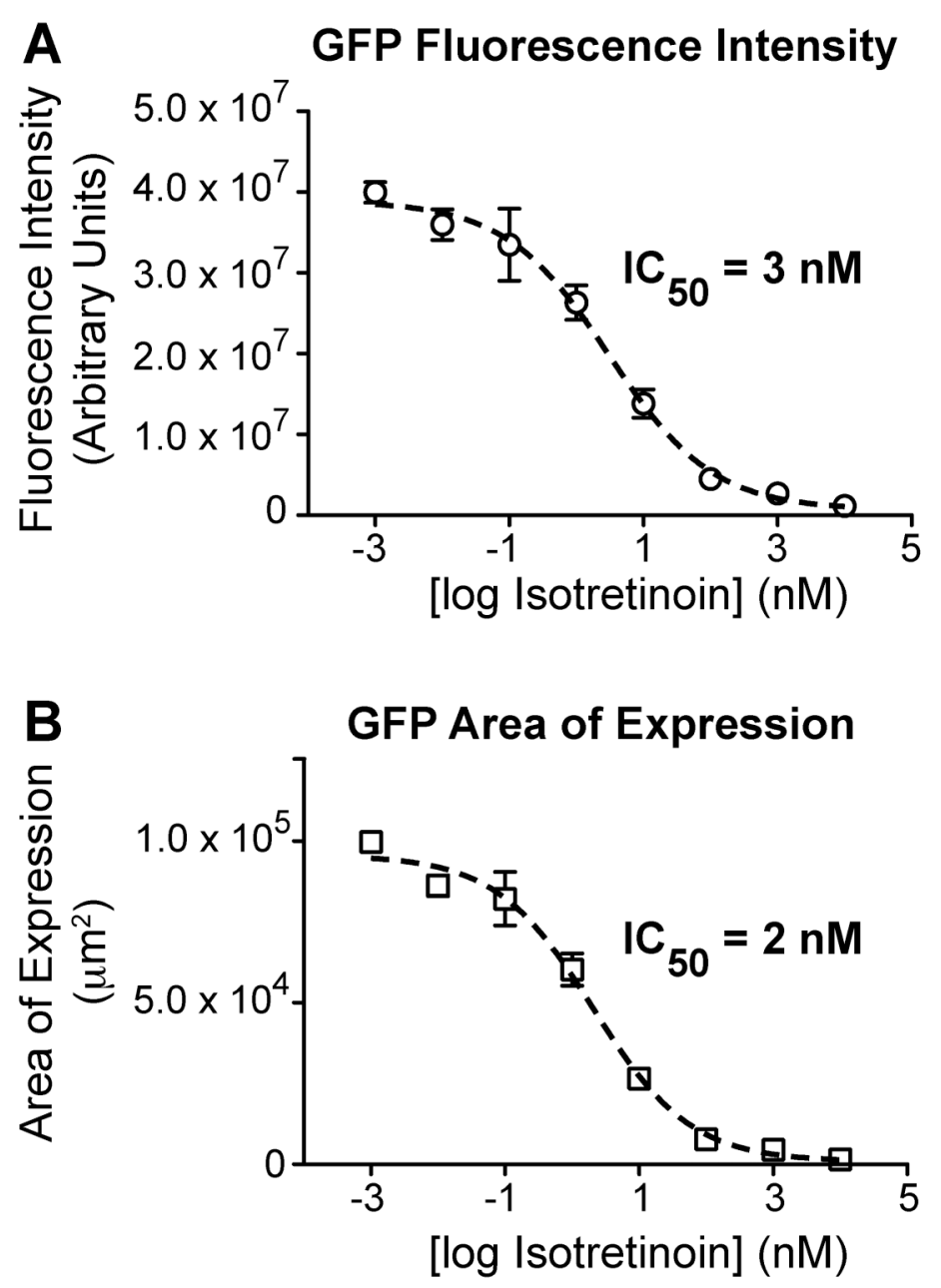

Fig. (6). A maximal projection of a reduced number of Z-planes can be used as a whole-spheroid mode of drug discovery. A) A dose response for isotretinoin measuring global CK5Pro-GFP-T47D spheroid expression using fluorescence intensity. B) Likewise, a dose response for isotretinoin using area of GFP expression $\left(\mu \mathrm{m}^{2}\right)$.

Harmony and Columbus software packages (Perkin Elmer). The first step in any image analysis algorithm is image segmentation, so that regions of interest can be divided, or segmented, according to various phenotypes. In a typical high content analysis algorithm, the initial image segmentation is done on stained nuclei, and current off-the-shelf analysis software will do a good job of segmenting individual cells based on the nuclear stain alone or nuclear stain coupled with a cytoplasmic stain. This proves to be problematic using a three-dimensional approach, because the cytoplasm of a cell is often in a different focal plane than the nucleus, leading to very inconsistent and misleading image segmentation. We overcame this problem by taking a region-based approach to analysis rather than an individual cell-based approach (Fig. 4). Briefly, we use the analysis software to identify regions of increased green fluorescence relative to background, either in individual focal planes (Z-stacks), or using maximal-projection images, which combine the highest intensity values across all image planes. All green regions were tiled with spots of varying size, from individual pixels to cells or cell clusters with elevated CK5Pro-GFP expression. Spots were filtered based on their fluorescence intensities as well as their size. The CK5Pro-GFP expression thus selected is next summed across the spheroid area, and quan- tified both as fluorescence intensity values across the selected region(s), and as actual area of GFP expression $\left(\mu \mathrm{m}^{2}\right)$. This algorithm can easily be adapted for other fluorescent readouts, such as Red Fluorescent Protein and its various forms (tdTomato, mCherry), or other reporters besides CK5. Thus the algorithm, with minimal modifications, can be applied to any number of MCTS model systems to monitor upor down-regulation of phenotypic reporters in order to determine small-molecule efficacy in multi cellular structures. For example, the algorithm can be applied to show whether small molecules are having effects only on peripheral cells (outer cell layers) or on entire spheroids: In one modification to the algorithm (data not shown), we can separate the spheroid into concentric rings based on the nuclear stain of the entire spheroid, and monitor effects on GFP expression in concentric regions, from the center to the periphery.

Finally, to demonstrate the robustness and utility of the MCTS system we have developed, we show that Z-prime values are greater than 0.5 for a number of output parameters from the analysis algorithm (Fig. 5). We also show that a dose-response curve can be generated using the same parameters and a range of isotretinoin concentrations in live MCTS (Fig. 6). The $\mathrm{IC}_{50}$ values for isotretinoin (Fig. 6) vary 
slightly depending on the parameter used (2-3 nM), but are consistent with our previously reported $\mathrm{IC}_{50} 0.8 \mathrm{nM}$ for blocking P4-mediated CK5Pro-GFP expression using the trans isomer (retinoic acid) in monolayer cell culture [38].

\section{CONCLUSION}

The MCTS model represents a robust in vitro 3D tumor model that can be used to bridge the gap of information between 2D-cell based assays and in vivo models of human tumors. Although the MCTS model is amenable to HTS drug discovery significant hurdles exist that have restricted its use to low-throughput cancer drug discovery. Nevertheless, MCTS HTS assays have been developed for endpoint detection but yielding limited information. However, the MCTS model offers researchers a "systems biology" approach to cancer drug discovery miniaturized into a single assay encompassing aspects of the tumor microenvironment and heterotypic tumor cell culture. Despite the challenges that remain with such systems, we demonstrate in this report a significant advance in achieving this using high-content image based algorithms to measure local and global complex phenomena in live MCTS. We show that acquiring data in this manner can be done in real time and is statistically robust for primary high-content screening cancer drug discovery and development.

\section{CONFLICT OF INTEREST}

The authors confirm that this article content has no conflicts of interest.

\section{ACKNOWLEDGEMENTS}

We would like to acknowledge the High Throughput and High Content Screening Core Facility at the University of Colorado AMC Skaggs School of Pharmacy and Pharmaceutical Sciences, and the Advanced Light Microscopy Core Facility, University of Colorado AMC. This work was funded in part by grants from the Cancer League of Colorado (to C.A.S. [principal investigator (PI)] and D.V.L. [co-PI]), the National Institutes of Health (NCI R01 CA140985; C.A.S. [PI], and D.V.L. [co-I]), and the Department of Defense Peer Reviewed Cancer Research Program career development award (CA120261, D.V.L.).

\section{SUPPLEMENTARY MATERIAL}

Supplementary material is available on the publishers website along with the published article.

\section{REFERENCES}

[1] Sutherland RM, Inch WR, McCredie JA, Kruuv J. A multicomponent radiation survival curve using an in vitro tumour model. Int J Radiat Biol Relat Stud Phys Chem Med 1970; 18: 491-5.

[2] Sutherland RM, McCredie JA, Inch WR. Growth of multicell spheroids in tissue culture as a model of nodular carcinomas. J Natl Cancer Inst 1971; 46: 113-20.

[3] Durand RE, Sutherland RM. Dependence of the radiation response of an in vitro tumor model on cell cycle effects. Cancer Res 1973; 33: 213-9.

[4] Frankel A, Buckman R, Kerbel RS. Abrogation of taxol-induced G2-M arrest and apoptosis in human ovarian cancer cells grown as multicellular tumor spheroids. Cancer Res 1997; 57: 2388-93.
[5] LaBarbera DV, Reid BG, Yoo BH. The multicellular tumor spheroid model for high-throughput cancer drug discovery. Expert Opin Drug Dis 2012; 0: 1-12.

[6] Li Q, Chen C, Kapadia A, et al. 3D Models of epithelialmesenchymal transition in breast cancer metastasis: highthroughput screening assay development, validation, and pilot screen. J Biomol Screen 2011; 16: 141-54.

[7] Olive PL, Durand RE. Drug and radiation resistance in spheroids: cell contact and kinetics. Cancer Metast Rev 1994; 13: 121-38.

[8] Sutherland RM. Cell and environment interactions in tumor microregions: the multicell spheroid model. Science 1988; 240: 177-84.

[9] West GW, Weichselbaum R, Little JB. Limited penetration of methotrexate into human osteosarcoma spheroids as a proposed model for solid tumor resistance to adjuvant chemotherapy. Cancer Res 1980; 40: 3665-8.

[10] Santini MT, Rainaldi G. Three-dimensional spheroid model in tumor biology. Pathobiology 1999; 67: 148-57.

[11] Jacks T, Weinberg RA. Taking the study of cancer cell survival to a new dimension. Cell 2002; 111: 923-5.

[12] Pampaloni F, Reynaud EG, Stelzer EH. The third dimension bridges the gap between cell culture and live tissue. Nat Rev Mol Cell Biol 2007; 8: 839-45.

[13] Bissell MJ, Radisky DC, Rizki A, Weaver VM, Petersen OW. The organizing principle: microenvironmental influences in the normal and malignant breast. Differentiation 2002; 70: 537-46.

[14] Xu R, Boudreau A, Bissell MJ. Tissue architecture and function: dynamic reciprocity via extra- and intra-cellular matrices. Cancer Metast Rev 2009; 28: 167-76.

[15] Nelson CM, Bissell MJ. Of extracellular matrix, scaffolds, and signaling: tissue architecture regulates development, homeostasis, and cancer. Annu Rev Cell Dev Biol 2006; 22: 287-309.

[16] Zahir N, Weaver VM. Death in the third dimension: apoptosis regulation and tissue architecture. Curr Opin Genet Dev 2004; 14: 71-80.

[17] Ghosh S, Spagnoli GC, Martin I, et al. Three-dimensional culture of melanoma cells profoundly affects gene expression profile: a high density oligonucleotide array study. J Cell Physiol 2005; 204: 522-31.

[18] Spencer VA, Xu R, Bissell MJ. Gene expression in the third dimension: the ECM-nucleus connection. J Mammary Gland Biol 2010; 15: 65-71.

[19] Hirschhaeuser F, Menne H, Dittfeld C, West J, Mueller-Klieser W, Kunz-Schughart LA. Multicellular tumor spheroids: an underestimated tool is catching up again. J Biotechnol 2010; 148: 3-15.

[20] Robertson FM, Ogasawara MA, Ye Z, et al. Imaging and analysis of 3D tumor spheroids enriched for a cancer stem cell phenotype. J Biomol Screen 2010; 15: 820-9.

[21] Rowehl RA, Crawford H, Dufour A, Ju J, Botchkina GI. Genomic analysis of prostate cancer stem cells isolated from a highly metastatic cell line. Cancer Genomics Proteomics 2008; 5: 301-10.

[22] Kitano H. Systems biology: a brief overview. Science 2002; 295: $1662-4$.

[23] Kreeger PK, Lauffenburger DA. Cancer systems biology: a network modeling perspective. Carcinogenesis 2010; 31: 2-8.

[24] Friedrich J, Seidel C, Ebner R, Kunz-Schughart LA. Spheroidbased drug screen: considerations and practical approach. Nat Protoc 2009; 4: 309-24.

[25] Hsiao AY, Tung YC, Qu X, Patel LR, Pienta KJ, Takayama S. 384 hanging drop arrays give excellent $Z$-factors and allow versatile formation of co-culture spheroids. Biotechnol Bioeng 2012; 109: 1293-304.

[26] Kunz-Schughart LA, Freyer JP, Hofstaedter F, Ebner R. The use of 3-D cultures for high-throughput screening: the multicellular spheroid model. J Biomol Screen 2004; 9: 273-85.

[27] le Roux L, Volgin A, Maxwell D, Ishihara K, Gelovani J, Schellingerhout D. Optimizing imaging of three-dimensional multicellular tumor spheroids with fluorescent reporter proteins using confocal microscopy. Mol Imaging 2008; 7: 214-21.

[28] Tung YC, Hsiao AY, Allen SG, Torisawa YS, Ho M, Takayama S. High-throughput 3D spheroid culture and drug testing using a 384 hanging drop array. Analyst 2011; 136:473-8.

[29] Lim E, Vaillant F, Wu D, et al. Aberrant luminal progenitors as the candidate target population for basal tumor development in BRCA1 mutation carriers. Nat Med 2009; 15: 907-13. 
[30] Bocker W, Moll R, Poremba C, et al. Common adult stem cells in the human breast give rise to glandular and myoepithelial cell lineages: a new cell biological concept. Lab Invest 2002; 82: 73746.

[31] Abd El-Rehim DM, Pinder SE, Paish CE, et al. Expression of luminal and basal cytokeratins in human breast carcinoma. J Pathol 2004; 203: 661-71.

[32] Cheang MC, Voduc D, Bajdik C, Leung S, et al. Basal-like breast cancer defined by five biomarkers has superior prognostic value than triple-negative phenotype. Clin Cancer Res 2008; 14: 1368-76.

[33] Livasy CA, Karaca G, Nanda R, et al. Phenotypic evaluation of the basal-like subtype of invasive breast carcinoma. Mod Pathol 2006; 19: 264-71.

[34] Axlund SD, Yoo BH, Rosen RB, et al. Progesterone-inducible cytokeratin 5-positive cells in luminal breast cancer exhibit progenitor properties. Horm Canc 2013; 4: 36-49.

[35] Kabos P, Haughian JM, Wang X, et al. Cytokeratin 5 positive cells represent a steroid receptor negative and therapy resistant subpopulation in luminal breast cancers. Breast Cancer Res $\mathrm{Tr}$ 2011; 128: 45-55.
[36] Horwitz KB, Dye WW, Harrell JC, Kabos P, Sartorius CA. Rare steroid receptor-negative basal-like tumorigenic cells in luminal subtype human breast cancer xenografts. Proc Natl Acad Sci U S A 2008; 105: 5774-9.

[37] Horwitz KB, Sartorius CA. Progestins in hormone replacement therapies reactivate cancer stem cells in women with preexisting breast cancers: a hypothesis. J Clin Endocrinol Metab 2008; 93: 3295-8.

[38] Yoo BH, Axlund SD, Kabos P, et al. A High-Content Assay to Identify Small-Molecule Modulators of a Cancer Stem Cell Population in Luminal Breast Cancer. J Biomol Screen 2012.

[39] Zhang JH. A Simple Statistical Parameter for Use in Evaluation and Validation of High Throughput Screening Assays. J Biomol Screen 1999; 4: 67-73.

[40] Longley DB, Harkin DP, Johnston PG. 5-fluorouracil: mechanisms of action and clinical strategies. Nat Rev Cancer 2003; 3: 330-8.

[41] Clemons M, Danson S, Howell A. Tamoxifen ("Nolvadex"): a review. Cancer Treat Rev 2002; 28: 165-80.

[42] Osborne CK. Tamoxifen in the treatment of breast cancer. N Engl J Med 1998; 339: 1609-18.

Received: August 15, 2013

(C) Reid et al.; Licensee Bentham Open.

This is an open access article licensed under the terms of the Creative Commons Attribution Non-Commercial License (http://creativecommons.org/licenses/by-nc/3.0/) which permits unrestricted, non-commercial use, distribution and reproduction in any medium, provided the work is properly cited. 